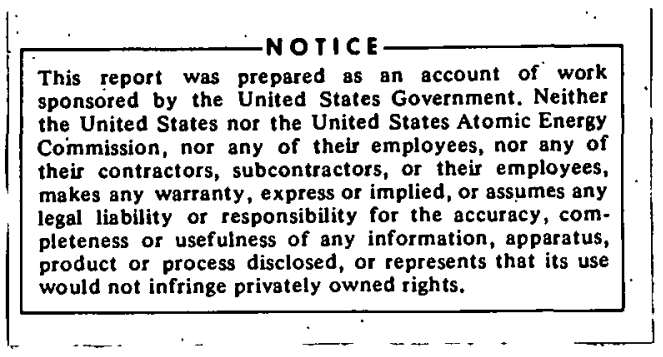

Report Number COO-2232-3

$$
\text { Conf- } 730320-2
$$

\title{
RESULTS ON LARGE TRANSVERSE MOMENTUM PHENOMENA
}

F.W. Büsser $\left.{ }^{*}\right)$, L. Camilleri, L. Di Lella, G. Gladding ${ }^{*}$ ),

A. Placci, B.G. Pope, A.M. Smith, J.K. Yoh and E. Zavattini

CERN, Geneva, Switzerland 3003000
I 0152431000

B.J. Blumenfeld $\mathrm{d}^{* * *}$ ) and L.M. Lederman $\begin{array}{ll}\text { Columbia University, NY, USA } & 2154000 \\ \text { IN/S } 1850000\end{array}$

R.L. Cool, L. Litt and S.L. Segler Rockefeller University, NY, USA

$$
\begin{aligned}
& 7228000 \\
& I N / S 5548000
\end{aligned}
$$

\footnotetext{
(Presented at the International Conference on

New Results from Experiments on High-Energy Particle Collisions, Vanderbilt University, Nashville, 26-28 March, 1973)
}

Geneva, 13 April 1973

*) On leave from II. Institut für Experimentalphysik, Hamburg, Germany.

$* *$ ) Visitor to CERN from the University of Illinois, USA.

***) N.S.F. Predoctoral Fellow. 


\section{DISCLAIMER}

This report was prepared as an account of work sponsored by an agency of the United States Government. Neither the United States Government nor any agency Thereof, nor any of their employees, makes any warranty, express or implied, or assumes any legal liability or responsibility for the accuracy, completeness, or usefulness of any information, apparatus, product, or process disclosed, or represents that its use would not infringe privately owned rights. Reference herein to any specific commercial product, process, or service by trade name, trademark, manufacturer, or otherwise does not necessarily constitute or imply its endorsement, recommendation, or favoring by the United States Government or any agency thereof. The views and opinions of authors expressed herein do not necessarily state or reflect those of the United States Government or any agency thereof. 


\section{DISCLAIMER}

Portions of this document may be illegible in electronic image products. Images are produced from the best available original document. 
RESULTS ON LARGE TRANSVERSE MOMENTUM PHENOMENA

F.W. Büsser*), L. Camilleri, L. Di Lella, G. Gladding**), A. Placci, B.G, Pope, A.M. Smith, J.K. Yoh and E. Zavattini CERN, Geneva, Switzerland

B.J. Blumenfeld ${ }^{* * *}$ ) and L.M. Lederman

Columbia University, NY, USA

R.L. Cool, L. Litt and S.L. Segler

Rockefeller University, NY, USA

\begin{abstract}
Preliminary results of an experiment on large transverse momentum phenomena performed at the CERN-ISR at centre-of-mass energies of 52.7 and $44.8 \mathrm{GeV}$ are presented. The topics studied were the inclusive reaction $p+p \rightarrow \pi^{0}+$ "anything", where the $\pi^{0}$ was emitted around $90^{\circ}$ in the centre-of-mass system, $\left(\pi^{\circ} \pi^{\circ}\right)$ correlations, and the charged multiplicity associated with large transverse momentum $\pi^{0}$ 's. In addition, results of a search for electrons and electron pairs are included.
\end{abstract}

\title{
INTRODUCTION
}

The experimental arrangement is shown in Fig. 1. Two identical spectrometers to detect electrons and photons around $90^{\circ}$ were set up on either side of an ISR intersection region, one inside and one outside the ring. Each of the spectrometers consisted of four parts:

i) An array of 10 (horizontal) $\times 6$ (vertical) lead-glass total absorption Čerenkov counters, each $15 \times 15 \times 35 \mathrm{~cm}^{3}$, to measure the energy of electrons and photons.

ii) An array of $8 \times 2$ thin lead-glass counters each $15 \times 35 \times 7.3 \mathrm{~cm}^{3}$, placed in front of the large blocks to enable the separation of electrons and photons from hadrons on the basis of energy $10 \mathrm{ss}^{1}$.

iii) Three modules of wire spark chambers (a total of 10 gaps: four planes with horizontal wires, four planes with vertical wires, two planes with wires having an angle of $15^{\circ}$ to the vertical direction) to measure the direction of the charged particles.

iv) Two scintillation counter hodoscopes $A$ and $B$, used for triggering the apparatus, and one hodoscope $Z$, consisting of 10 counters each $6.5 \times 60 \times 1 \mathrm{~cm}^{3}$, to measure the ionization loss of the charged particles. A fourth set of counters was placed behind the lead-glass blocks to permit the selection of particles passing through the array for calibration of the lead-glass blocks.

For measuring the inclusive single $\pi^{0}$ spectrum a lead sheet of 0.25 radiation length was placed between the $Z$ hodoscope and the first wire spark chamber module. Photons from the $\pi^{0}$ decay could be identified from the lack of sparks in the three gaps of the first wire spark 
chamber module and from the appearance of many sparks in the other two modules. The pulse height in the $Z$ counter hodoscope behind the conversion point would normally correspond to more than one charged particle.

Two $1 \times 1 \mathrm{~m}^{2}$ large scintillation counters $\Sigma_{1}$ and $\Sigma_{2}$ surrounded the beam pipes downstream of the interaction region, and were requirod in coincidence to ensure that the event results from a beam-bean collision.

The energy calibration of the spectrometers is believed to be known to within $\pm 4 \%$. The solid angle subtended by each spectrometer was approximately $1 \mathrm{sr}$.

\section{RESULTS ON INCLUSIVE $\pi^{0}$ SPECTRA}

Since a coincidence between the two $\Sigma$ counters was required, the apparatus was only sensitive to $73 \%$ of the inelastic events at a centre-of-mass energy $\sqrt{\mathrm{s}}=52.7 \mathrm{GeV}$ and to $60 \%$ of the inelastic events at $\sqrt{s}=44.8 \mathrm{GeV}$. This means that the single production spectra which we obtained are not really inclusive and we shall define them as quasiinclusive. This $\Sigma_{1} \Sigma_{2}$-coincidence requicement may introduce some $s$ or $p_{1}^{*}$ dependent biases, which have not yet been evaluated in detai]. The measured spectra are shown in Fig. 2. For these spectra, only events where a gamma-ray was seen to convert in the lead vere considered. The data at $\sqrt{\mathrm{s}}=52.7 \mathrm{GeV}$ represent approximately $10^{10}$ inelastic pp collisions. The data at $\sqrt{\mathrm{s}}=52.7 \mathrm{GeV}$ can be adequately fitted by the following phenomenological formula

$$
E \frac{d^{3} \sigma}{d^{3} \vec{p}}=\frac{(2.16 \pm 0.22) \times 10^{-27}}{p_{1}^{*^{4}}} \times e^{-(1.38 \pm 0.02) \mathrm{p}_{\perp}^{*} \mathrm{~cm}^{2} / \mathrm{G} \varepsilon V \circ \mathrm{sr}}
$$

in the interval $2.6<p_{\perp}^{*}<7.9 \mathrm{GeV} / \mathrm{c}$, where $p_{\perp}^{*}$ is the txansverse momentum of the $\pi^{0}$ in the centre-of-mass system.

\section{RESULTS OF $\left(\pi^{0} \pi^{0}\right)$ CORRELATIONS}

Whenever $a \pi^{0}$ is observed in one detector in coincidence with a $\pi^{0}$ in the opposite detector, it is possjble to calculate the invariart mass of the $\left(\pi^{0} \pi^{0}\right)$ system using the formula (correct for high momenta)

$$
M_{\pi 0}^{2} \pi^{0}=4 p_{1} p_{2} \sin ^{2}(\alpha / 2),
$$

where $p_{1}$ and $p_{2}$ are the momenta of the two $\pi^{0}$ 's and $\alpha$ is the opening angle of the pair. We have restricted our analysis to those events for which $p_{1}$ and $p_{2}$ are both at least $2 \mathrm{GeV} / \mathrm{c}$. Figure 3 shows the. experimental distribution of $M_{\pi^{0} \pi^{0}}$ obtained at a centre-of-mass energy of $52.7 \mathrm{GeV}$ and for $1.5 \times 10^{9}$ 袋elastic interactions. These data are compared to the results of a calculation based on the folloring two assumptions: 
i) the probability of observing a $\pi^{0}$ in one detector is independent of the probability of observing another $\pi^{0}$ in the other detector,

ii) each $\pi^{0}$ has a momentum distribution given by the experimental distribution measured for single $\pi^{0}$ 's in the same experiment.

It is observed, that there is a strong correlation between the two $\pi^{0} \mathrm{~s}$. How much of it is just the effect of momentum conservation is not known at present.

\section{CHARGED MULTIPLICITIES. ASSOCIATED WITH LARGE TRANSVERSE MOMENTUM}

The average charged multiplicities on the same side as and the opposite side to a $\pi^{0}$ are shown in Fig. 4, plotted as a function of the centre-of-mass transverse momentum $\mathrm{p}_{+}^{*}$ of the $\pi^{0}$. When on the opposite side of the $\pi^{0}$, the multiplicity is seen to rise linearly, possibly as an effect of momentum conservation. Also shown (dotted line). are the charged multiplicities on the two sides for a $\Sigma_{1} \Sigma_{2}$ trigger not requiring a $\pi^{0}$.

The solid angles subtended by the spark chambers in the c.m.s. of the pp collisions are $\Omega_{\text {outside }}=1.33 \mathrm{sr}, \Omega_{\text {inside }}=0.79 \mathrm{sr}$ at $\sqrt{\mathrm{s}}=$ $=52.7 \mathrm{GeV}$. The average charged multiplicities associated with a $\pi^{0}$ with $\mathrm{p}_{\perp}^{*}>3 \mathrm{GeV} / \mathrm{c}$ in the inside detector are

$$
\begin{aligned}
& \left\langle\mathrm{n}_{\text {outside }}\right\rangle=0.98 \\
& \left\langle\mathrm{n}_{\text {inside }}\right\rangle=0.34 .
\end{aligned}
$$

For a $\Sigma_{1} \Sigma_{2}$ only trigger the corresponding numbers are

$$
\begin{aligned}
& \left\langle\mathrm{n}_{\text {outside }}\right\rangle=0.41 \\
& \left\langle\mathrm{n}_{\text {inside }}\right\rangle=0.20 .
\end{aligned}
$$

SEARCH FOR ELECTRONS

High transverse momentum single electrons from the reaction $p+p \rightarrow e^{-}+$"anything" would be expected from the leptonic decay of - charged intermediate bosons, or more generally from the leptonic decay of any not yet discovered heavy particles. Electrons were identified by requiring a track to be observed in the wire spark chamber planes, coming from the interaction region and triggering the three scintillation counter hodoscopes. Tracks giving an energy loss $\mathrm{dE} / \mathrm{dx}>1.6$ times minimum ionization in the hodoscope $Z$ were rejected, to discriminate against narrow angle electron pairs originating from photons converting in the ISR vacuum pipe or from Dalitz pairs. The spectrum of the observed events is shown in Fig. 5. These data represent approximately $7 \times 10^{9}$ interactions at $\sqrt{\mathrm{s}}=52.7 \mathrm{GeV}$. This spectrum represents an upper limit for electron production, since a large fraction, if not all of the events, may be due to background. 
Special runs performed at the CERN Proton Synchrotron have indicated that the fraction of charged pions that would satisfy the electron requirements is approximately 1/300. Photon conversion in the ISR vacuum pipe would account for about $1 / 1000$ of the observed neutral pion spectrum.

The most serious backgrounds are (i) chance overlap in space, within the angular acceptance of one lead-glass block, of a charged pion satisfying the track requirement and a neutral pion satisfying the energy requirement, and (ii) charged pions which interact in the lead-glass. Studies are at present under way to estimate the magnitude of this background.

Electrons from the decay of charged $W^{\prime}$ s would produce a sharply peaked spectrum with transverse momenta approximately half of the mass of the $W$, if the $W$ is produced at rest. The precise momentum distribution is dependent on the particular production model assumed for the W. In Fig. 5 two examples of the expected electron momentum distributions are shown for different masses of the $W$ assuming the parton model of Dreli and $\mathrm{Yan}^{2}$ and a value of $B \cdot \sigma_{W}=10^{-33} \mathrm{~cm}^{2}$, where $B$ is the branching ratio $B=W \rightarrow e v / W \rightarrow$ all and $\sigma_{W}$ the total production cross-section for $W$.

The following results were obtained:

i) one event $/(\mathrm{GeV} / \mathrm{c})$ corresponds to a detected cross-section $\sigma_{\mathrm{det}}=$ $=5.1 \times 10^{-36} \mathrm{GeV} / \mathrm{c}\left(7 \times 10^{9}\right.$ interactions, $\left.\sigma_{\text {inel }}=35.6 \mathrm{mb}\right)$,

ii) no events were seen with $p_{\perp}^{*}>5.3 \mathrm{GeV} / \mathrm{c}$,

iii) the estimated efficiency is about $6 \%$, .

iv) with $95 \%$ confidence level it is estimated that $B \cdot \sigma_{W}<$ $<2.6 \times 10^{-34} \mathrm{~cm}^{2}$ for masses greater than $11 \mathrm{GeV}$.

$$
\text { STUDY OF THE REACTION } \mathrm{p}+\mathrm{p} \rightarrow \mathrm{e}^{+} \mathrm{e}^{-}+\text {"ANYTHING" }
$$

In this case the effective mass is measured directly and the experiment is sensitive to cross-section branching ratios of the order of $10^{-34} \mathrm{~cm}^{2}$ for neutral $W^{\prime} s$ and heavy vector mesons.

The analysis of the electron pair spectra is still in progress, but at this stage the following conclusions can be stated. A small number of candidates has been found. It is believed that many of these can be attributed to the background processes mentioned above. No obvious resonant structure has been observed; no event with an invariant mass greater than $5 \mathrm{GeV} / \mathrm{c}^{2}$ has been observed. As an indication of the sensitivity of this experiment the integrated electron pair cross-section-observed in $1.07 \times 10^{10}$ inelastic interactions is displayed in Fig. 6 as a function of the lower mass limit of the integration. The $95 \%$ confidence level line represents the absence of three events in the high mass region. The prediction of the parton model of Drell and $\mathrm{Yan}^{2}$, which has been normalized to the dimuon data of Christenson et $a 1^{3}$, is shown. The curve labelled $\sigma_{B} 0$ is the enhancement of the Drel1 and Ýan model expected to be shown by the heavy photon pole of Lee and Wi.ck ${ }^{4}$. 
REFERENCES

1. B.J. Blumenfeld, L.M. Lederman, R.L. Cool and S.L. Segler, Nuclear Instrum. Methods 97, 42.7 (1971).

2. S.D. Drell and T.M. Yan, Phys. Rev. Letters 25, 316 (1970).

3. J.H. Christenson, G.S. Hicks, L.M. Lederman, P.J. Lipmán, B.G. Pope and E. Zavattini, Phys. Rev. Letters 25, 1523 (1970).

4. T.D. Lee and G.C. Wick, Phys. Rev. D2, 1033 (1970). 
CERN - COLUABIA - ROCKEFELLER

COLLABORATION

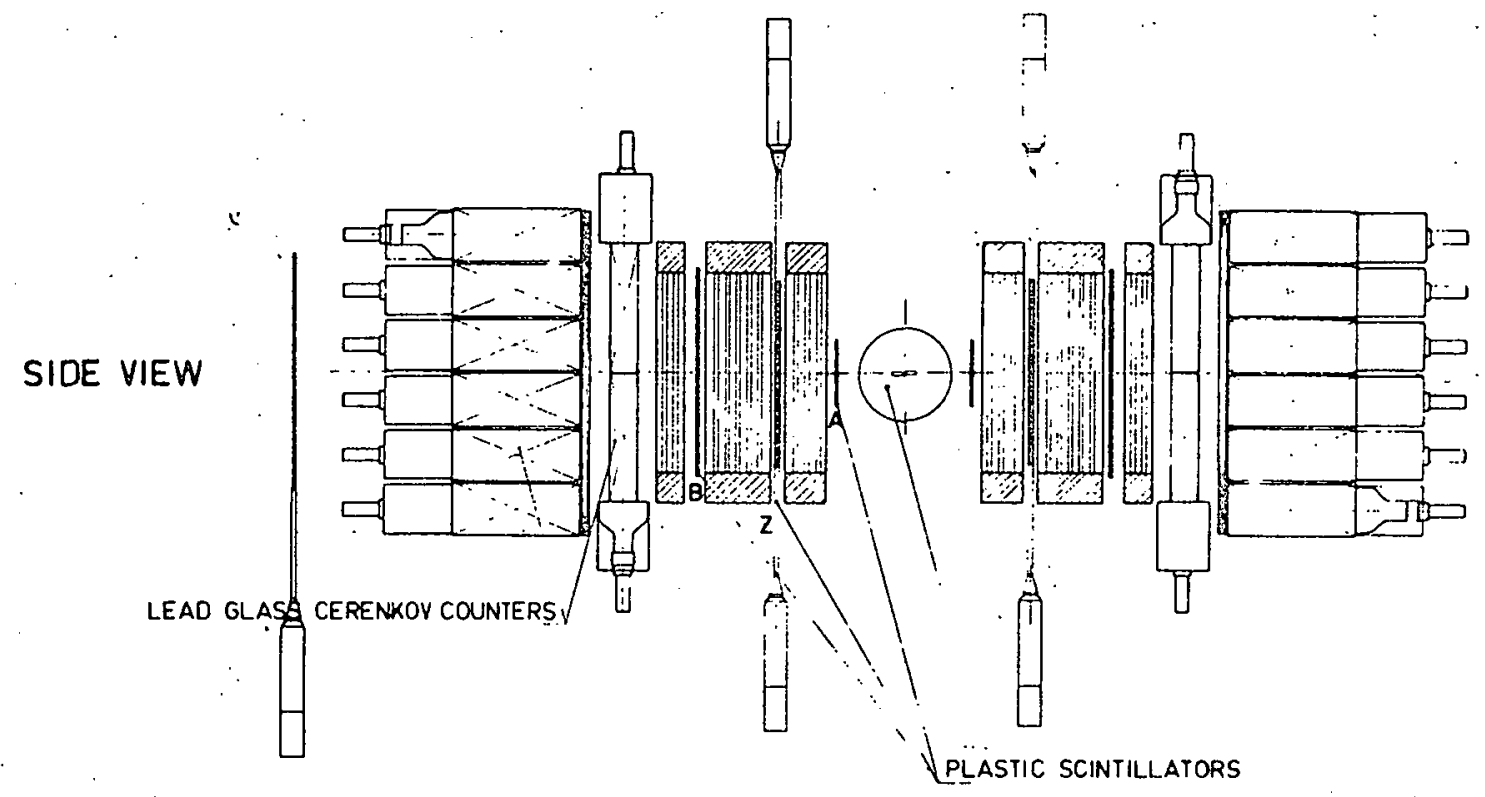

TOP VIEW

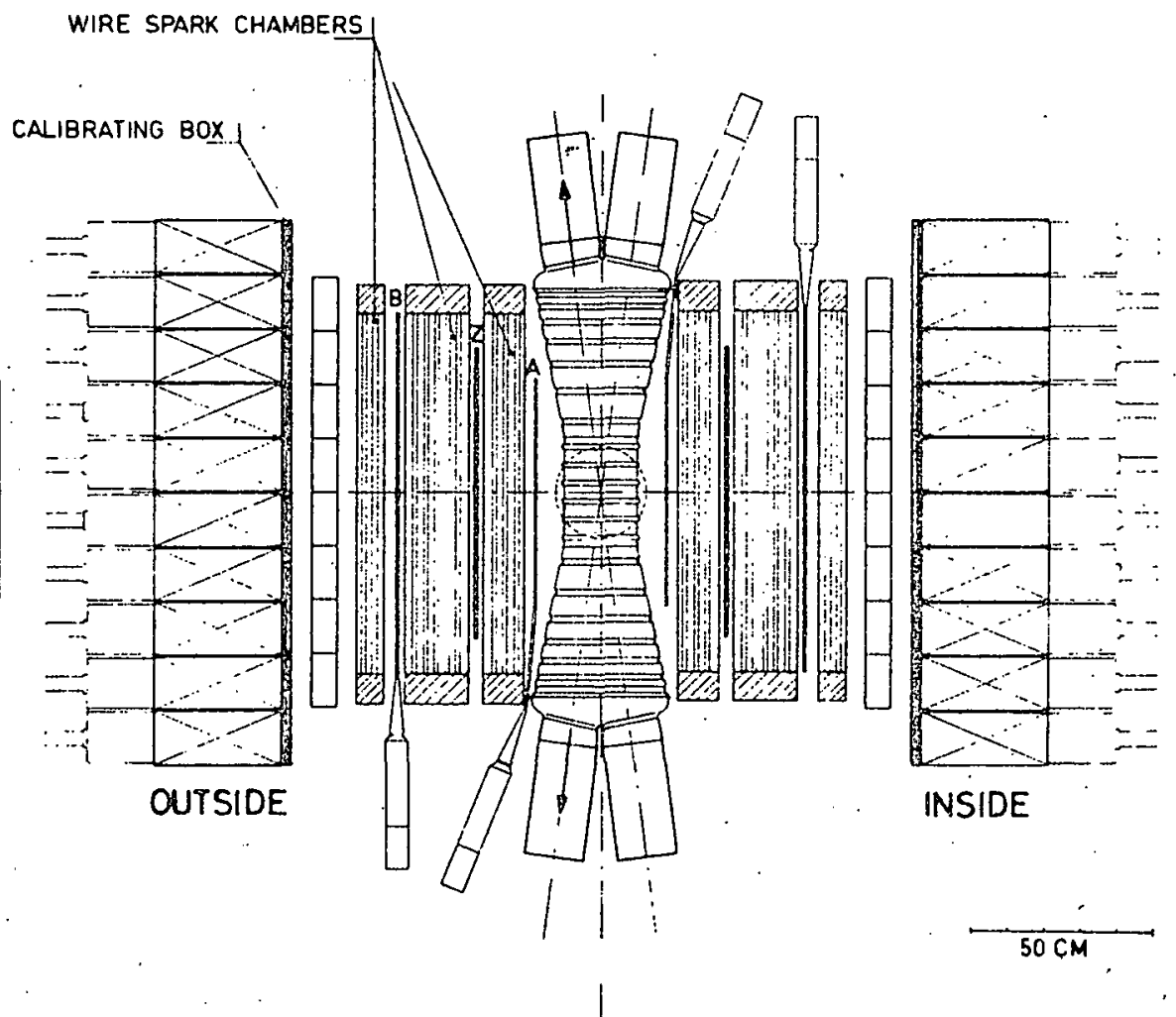

Fig. 1 Experimental arrangement 


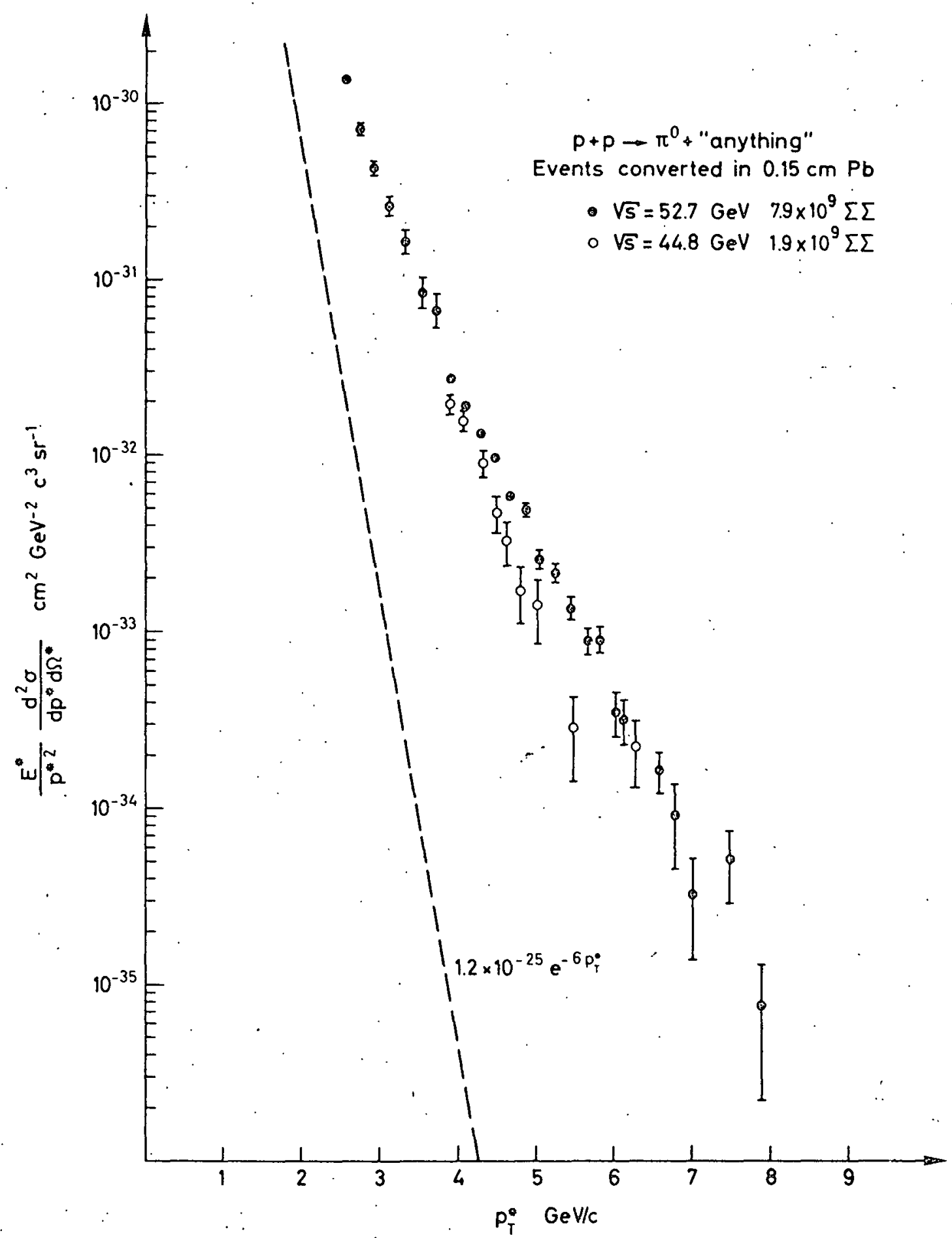

Fig. 2 Invariant cross-section of the inclusive reaction $\mathrm{p}+\mathrm{p} \rightarrow$ $\rightarrow \pi^{0}+$ "anything" at centre-of-mass energies of $\sqrt{\mathrm{s}}=52.7$ and $\sqrt{\mathrm{s}}=$ $=44.8 \mathrm{GeV}$, as a function of the transverse momentum $p_{1}^{*}$ of the $\pi^{0}$ in the centre-of-mass system. Also shown (dotted line) is the extrapolation of lower $\mathrm{p}_{\perp}^{*}$ data. 


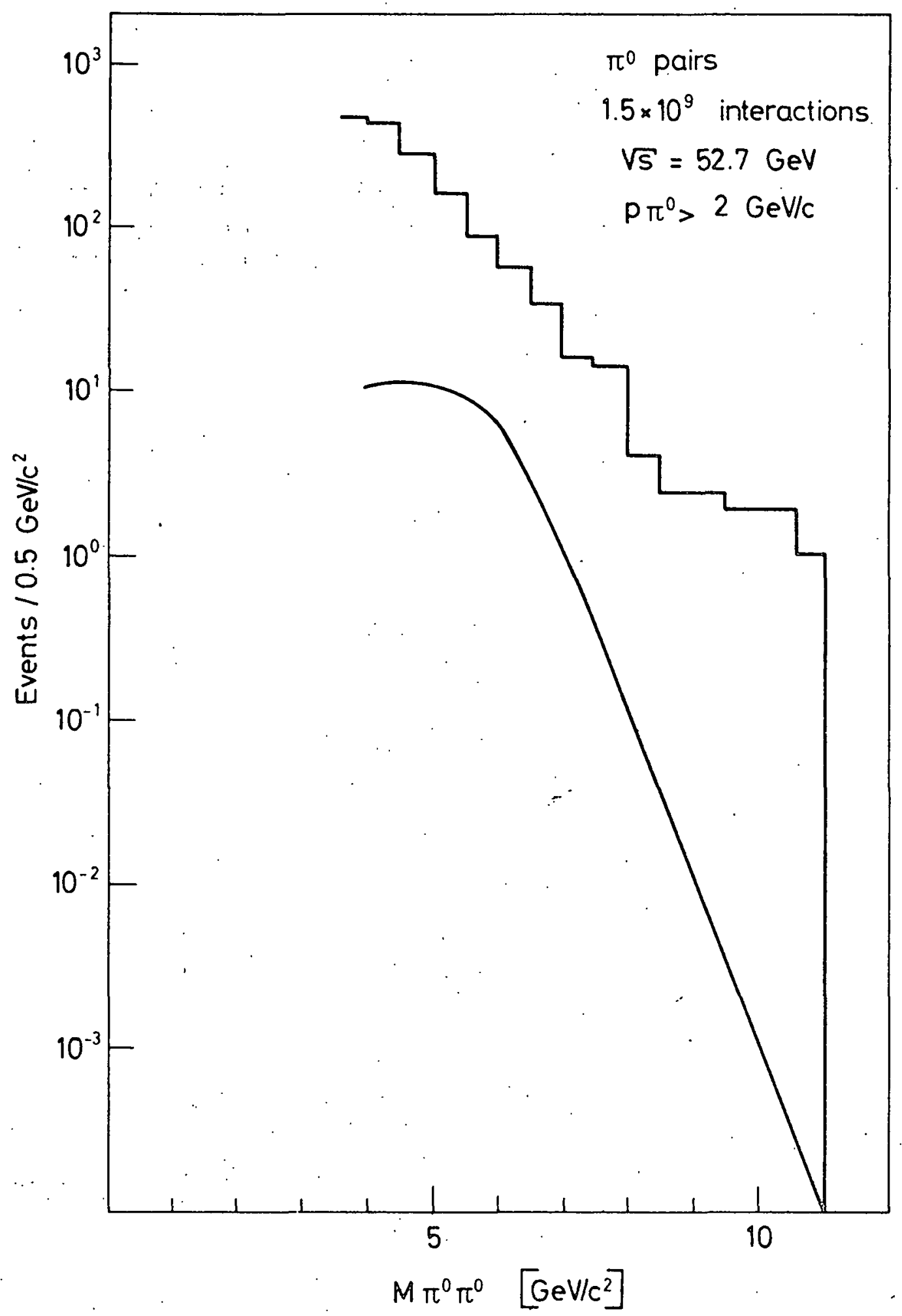

Fig. 3 Experimental distribution of the invariant mass of the $\left(\pi^{0} \pi^{0}\right)$ spectrum at $\sqrt{\mathrm{s}}=52.7 \mathrm{GeV}$. The solid line is the prediction of uncorrelated $\pi^{0}$ 's. 
ASSOCIATED MULTIPLICITIES

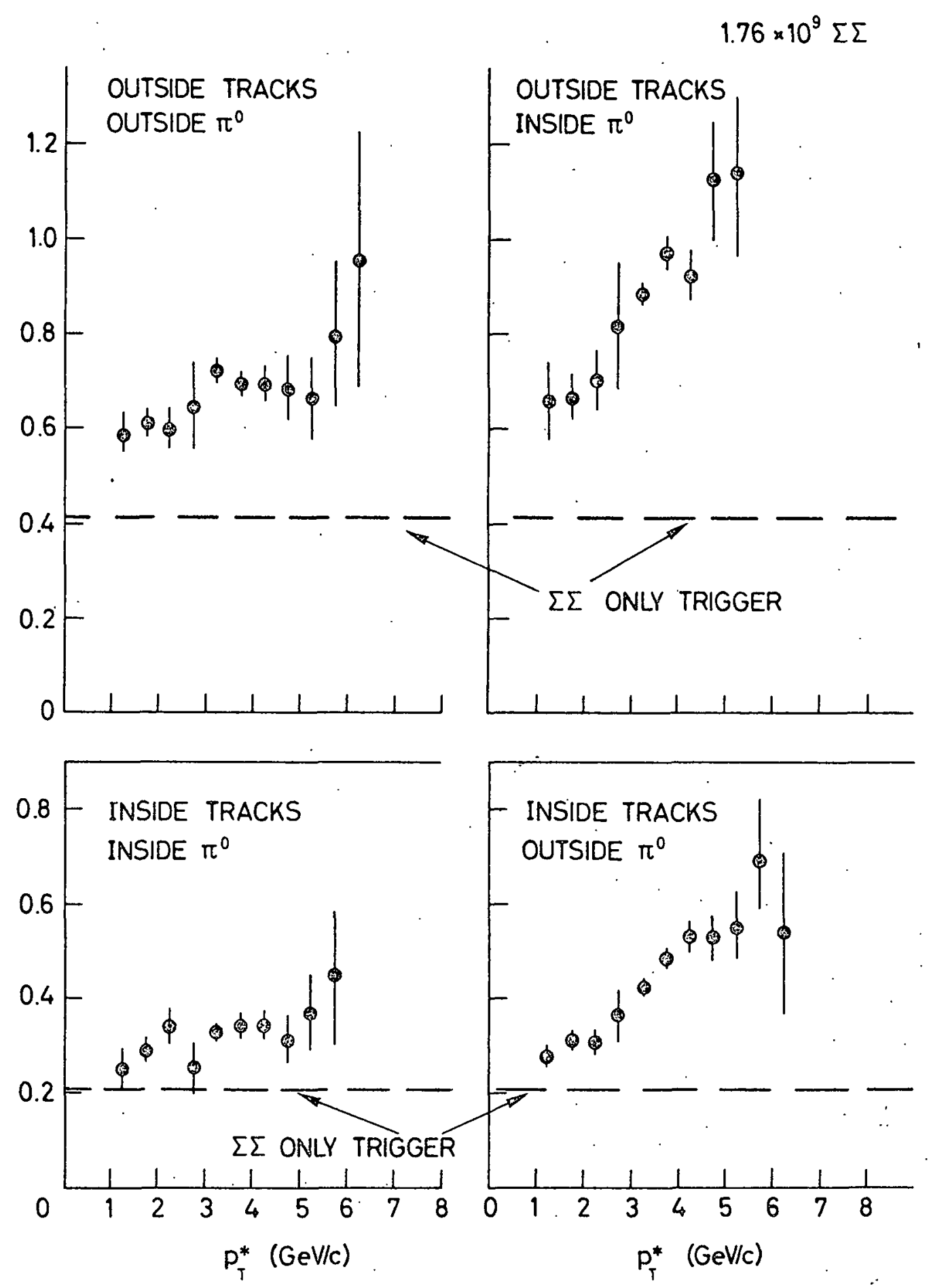

Fig. 4 . Average charged multiplicities. on the same side as and opposite side to a $\pi^{0}$, as a function of the centre-of-mass transverse momentum $\mathrm{p}_{1}^{*}$ of the $\pi^{0}$. Also shown (dotted lines) are the charged multiplicities for a $\sum \sum$ trigger not requiring a $\pi^{0}$. 


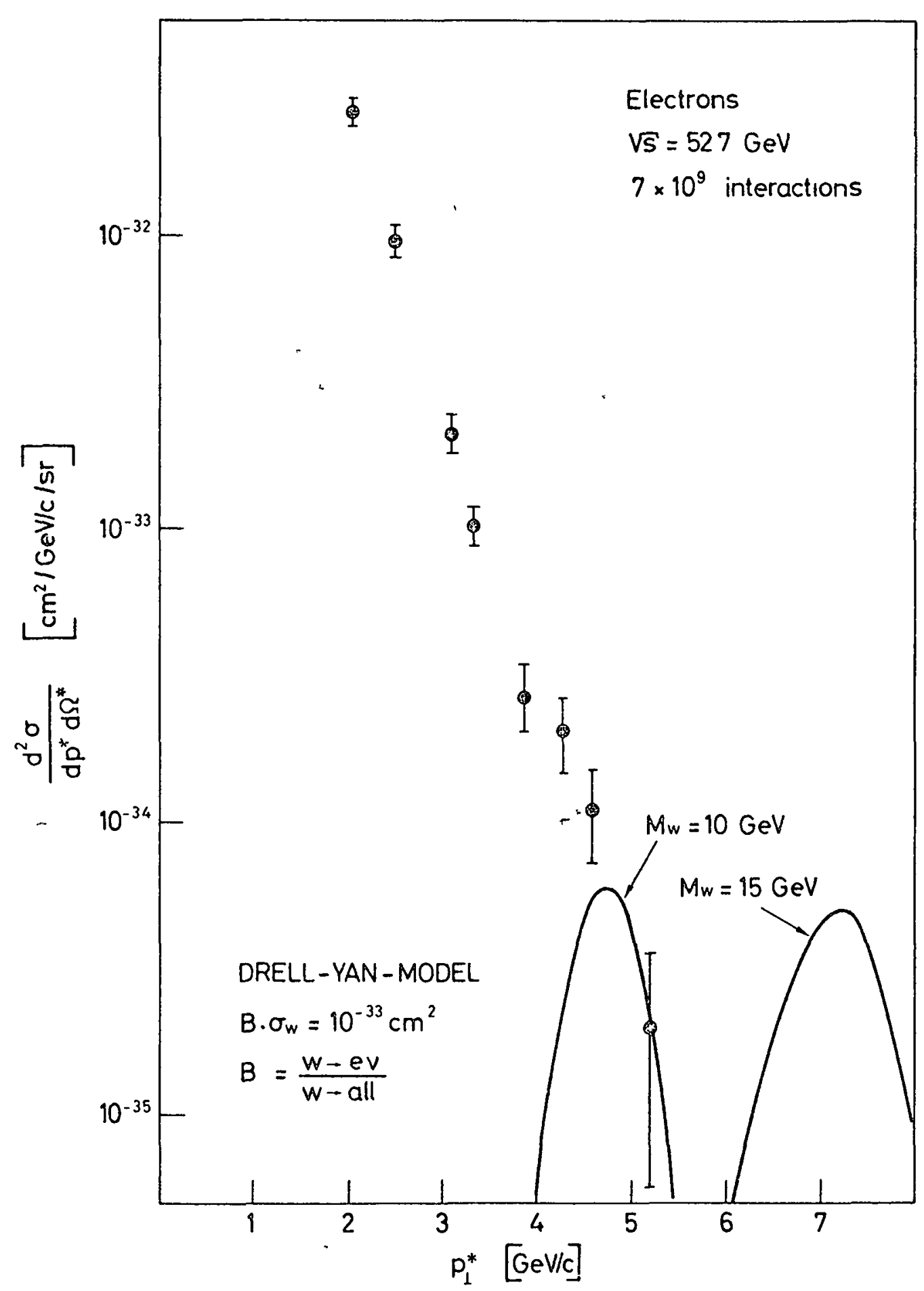

Fig. 5 Electron spectrum at $\sqrt{s}=52.7 \mathrm{GeV}$. In addition two expected electron momentum distributions for different masses of the $W$ (intermediate boson) are shown (solid lines) assuming the parton model of Drell and $Y$ ar $r_{1}$ and a value of $B \cdot \sigma_{W}=10^{-33} \mathrm{~cm}^{2}$. 


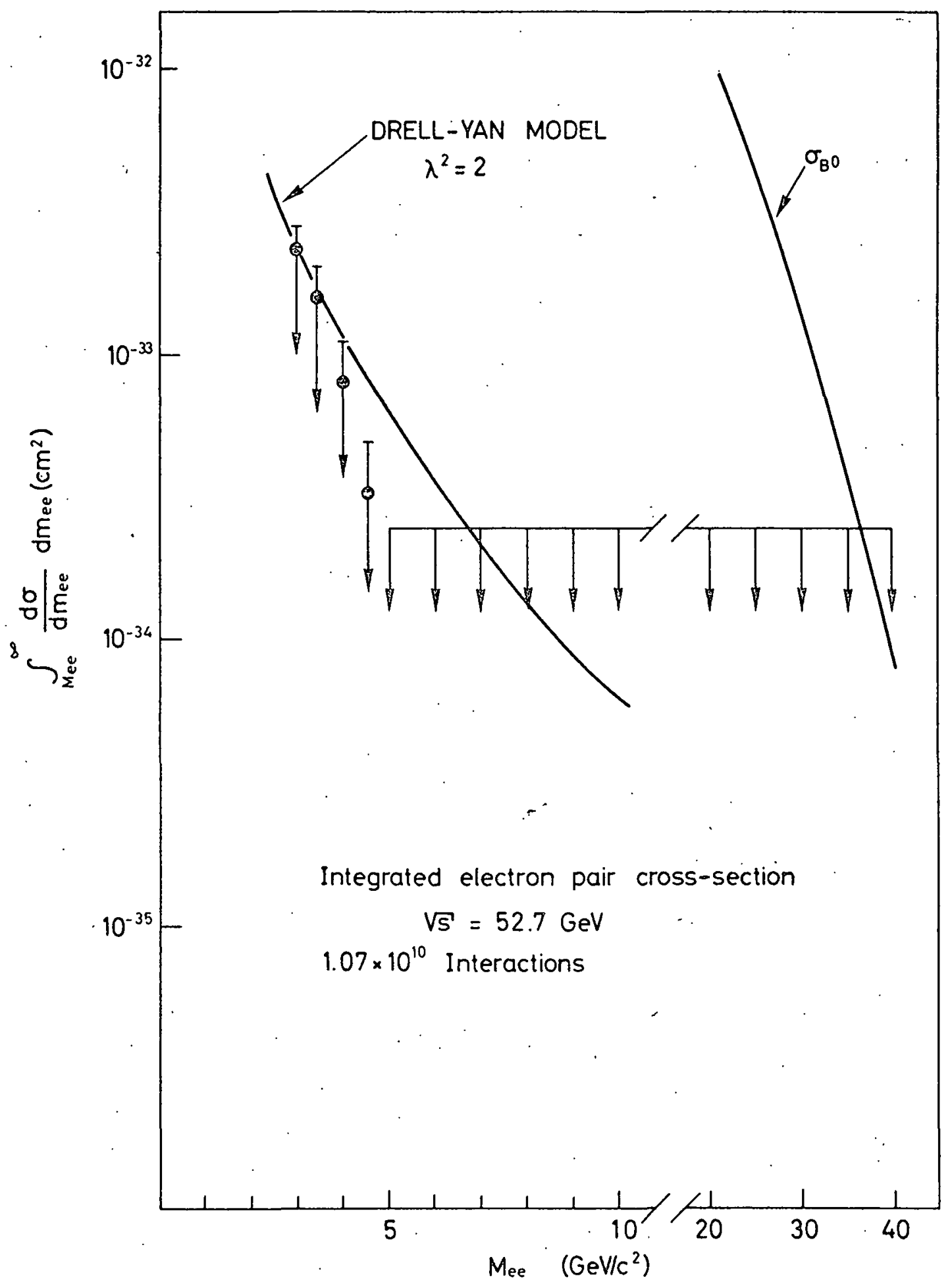

Fig. 6 Integrated electron pair cross-section as a function of the lower mass limit. Also shown are the predictions of the parton model of Drell and $\mathrm{Yan}^{2}$ and of the model of Lee and Wick ${ }^{4}$ (curve labelled $\left.\sigma_{B^{0}}\right)$. The spectrum presented is only an upper limit. 